\title{
Le rôle des personnages dans les rouages de l'intrigue ${ }^{1}$
}

\author{
O papel dos personagens na dinâmica do enredo
}

The role of characters in plot dynamics

Raphaël Baroni

Université de Lausanne - Lausanne - Suíça

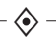

\begin{abstract}
Résumé: Cet article propose une synthèse d'un ouvrage qui pose les fondements d'une approche narratologique d'orientation stylistique et rhétorique visant à décrire avec le plus de précision possible le mécanisme des récits littéraires immersifs et intrigants. Partant du constat de l'existence d'une forte polysémie autour de la notion d'intrigue, je proposerai de partir d'une définition fonctionnelle apte à souligner le rapport étroit entre la dynamique du récit et la mise en intrigue, qui noue, entretien et dénoue une tension dans l'expérience esthétique. J'évoquerai également la manière dont la caractérisation des personnages et la mutabilité de leurs rôles peuvent déterminer de manière essentielle le profil de la tension narrative.
\end{abstract}

Mots clés: Intrigue; Tension narrative; Personnage; Narratologie; Rhétorique; Stylistique

Resumo: Este artigo fornece um resumo de um livro que estabelece as bases para uma abordagem estilística e retórica da narratologia cujo objetivo é descrever com a maior precisão possível o mecanismo de narrativas literárias imersivas e intrigantes. Observando a existência de uma forte polissemia em torno da noção de trama, proponho começar com uma definição funcionalista capaz de enfatizar a estreita relação entre a dinâmica narrativa e o empenho, que constrói, mantém e resolve a tensão na experiência estética. Eu também menciono como a caracterização dos personagens e a mutabilidade de seus papéis podem determinar de forma essencial o perfil da tensão narrativa.

Palavras-chave: Trama; Tensão narrativa; Caráter; Narratologia; Retórica; Estilística

\begin{abstract}
This article provides a summary of a book that lays the foundation for a stylistical and rhetorical approach of narratology whose aim is to describe with the greatest possible precision the mechanism of immersive and intriguing literary narratives. Noting the existence of a strong polysemy around the notion of plot, I propose to start with a functionalist definition capable to emphasize the close relationship between narrative dynamics and emplotment, which builds, maintain, and resolve tension in the aesthetic experience. I also mention how the characterization of characters and the mutability of their roles can determine in an essential way the profile of narrative tension.
\end{abstract}

Keywords: Plot; Narrative tension; Character; Narratology; Rhetorics; Stylistics

\section{Les avatars de l'intrigue: polysémie, double séquence et fonctions discursives}

De nos jours, le concept d'intrigue circule largement parmi différentes communautés de chercheurs et dans les institutions scolaires, sans que l'on sache si l'on parle toujours du même objet. Cette polysémie a été

\footnotetext{
1 Cet article reprend certains arguments centraux de mon prochain livre, Les Rouages de l'intrigue, à paraître aux éditions Slatkine au printemps 2017.
}

accentuée ces dernières années par le fait que ce terme, autrefois réservé au domaine de la poétique littéraire, s'est répandu bien au-delà de son périmètre d'origine. Pour certains, les récits historiques, les nouvelles véhiculées par la presse quotidienne, voire les narrations conversationnelles les plus spontanées, ou les nombreux avatars du storytelling posséderaient des intrigues. Dans un mouvement inverse, d'autres dressent le constat que la modernité aurait abouti, au cours du $\mathrm{XX}^{\mathrm{e}}$ siècle, à la progressive disparition de cette forme d'organisation du roman, jugée trop conventionnelle ou commerciale par les 
avant-gardes littéraires. On ajoutera que le sens commun nous pousserait plutôt à postuler l'inverse, à savoir que les intrigues les plus saillantes, c'est-à-dire celles qui se signalent à nous parce qu'elles parviennent à nouer puis à dénouer une tension lors de notre progression dans le récit, se rencontrent rarement dans les textes historiques, journalistiques ou conversationnels, alors qu'elles abondent dans les fictions, quels que soient les médias, les genres ou les périodes historiques envisagés. Si l'on adopte ce point de vue, ce qui apparaît crucial, c'est le lien qui peut être établi entre la mise en intrigue et l'art de raconter les histoires passionnantes. Or, ces intrigues saillantes, qui reposent sur l'entretien d'un mystère ou d'un suspense jusqu'au dénouement, apparaissent bien loin de la configuration rationnelle des comptes rendus historiques ou journalistiques, dont la fonction est davantage d'expliquer le passé que d'intriguer le lecteur. Nous constatons que nous nageons alors en pleine confusion...

En réalité, il est probablement vain de chercher un sens unique à l'intrigue. Il vaut mieux commencer par reconnaître l'existence d'une polysémie au sein de laquelle différents usages spécialisés s'opposent entre eux, et s'opposent également, le plus souvent, aux usages véhiculés par le langage ordinaire ${ }^{2}$. Ainsi que le résume l'entrée "plot" de la Routledge Encyclopedia of Narrative Theory:

\begin{abstract}
Malgré l'apparente simplicité de l'objet auquel elle se réfère, l'intrigue est l'un des termes les plus insaisissables de la théorie du récit. Les narratologues l'utilisent pour se référer à une variété de phénomènes différents. La plupart des définitions de base du récit buttent sur la question de la séquentialité, et les tentatives répétées de redéfinir les paramètres de l'intrigue reflètent à la fois la centralité et la complexité de la dimension temporelle du récit (DANNENBERG, 2005 , p. 435 , m.t.).
\end{abstract}

Hans Porter Abbott dresse un constat similaire et dénombre quant à lui au moins trois sens différents pour ce concept: 1) l'intrigue représenterait la trame de l'histoire, quelque chose qui pourrait être rapproché du concept de fabula;2) l'intrigue se réfèrerait à la manière dont le récit se départit de l'ordre chronologique de l'histoire, ce qui la ferait correspondre avec le concept formaliste de sujet; 3) l'intrigue serait enfin "la combinaison d'une sélection et d'un séquençage des événements, qui fait qu'une histoire est ce qu'elle est et non un simple matériau brut", cette opération la transformant en un "tout intelligible" (2007, p. 44, m.t.). De manière à réduire les problèmes liés à

\footnotetext{
Sur le sens de l'intrigue, notamment dans son étymologie et dans ses usages dans la langue française, je renvoie à l'essai fondamental de Johanne Villeneuve (2004)
}

cette polysémie, Abbott adresse ses recommandations: il affirme que si le premier des trois usages est celui qui demeure le plus proche de la manière dont on se sert ordinairement du terme anglais "plot", les deuxième et troisième pourraient être désignés de manière plus adéquate par le terme "emplotment", dont l'équivalent en français serait "mise en intrigue" (ABBOTT, 2007, p. 44 , m.t.).

On pourrait cependant objecter à Abbott que son inventaire demeure incomplet. Il affirme en effet que les lecteurs formulent souvent des évaluations du type: "c'était ennuyeux, il n'y avait pas d'intrigue" (2007, p. 44 , m.t.) et il en tire la conclusion, à mon avis erronée, que dans son usage ordinaire, le mot "intrigue" ("plot") renvoie à l'histoire. On pourrait au contraire en déduire que les lecteurs se réfèrent ici plus spécifiquement au manque d'intérêt de cette histoire, à l'absence de mystère, de suspense ou de surprise qui la caractérise, ce qui met également en cause la manière dont cette histoire est racontée et la fonction de l'intrigue dans l'interaction entre texte et lecteur, non une hypothétique absence d'histoire. D'ailleurs, dans le Living Handbook of Narratology, Karin Kukkonen signale qu'il existe deux significations fonctionnelles qui viennent s'ajouter à la définition formaliste de l'intrigue en tant que structure globale de l'histoire racontée:

(1) L'intrigue comme structure fixe et globale, quand on considère la configuration de l'ensemble des événements qui forment la trame de l'histoire, du début, du milieu et de la fin.

(2a) L'intrigue comme structuration progressive, quand on considère les connections entre les événements de l'histoire, leurs causes et leurs conséquences, telles que les lecteurs les perçoivent.

(2b) L'intrigue en tant que planification de l'auteur, quand on considère la manière dont ce dernier structure le récit en vue de produire des effets particuliers (KUKKONEN, 2014, p. 706).

On peut essayer de tirer une synthèse de ces usages divergents du terme, tout en restreignant son périmètre de manière éviter tout malentendu ultérieur. Dans le cas le plus élémentaire, on s'est servi de ce terme pour désigner l'organisation séquentielle globale des événements racontés, c'est-à-dire la trame de l'histoire, son organisation temporelle et causale telle qu'elle peut être reconstruite lorsque le récit est achevé. À un deuxième niveau, ce terme peut renvoyer à l'agencement séquentiel de cette histoire par le "discours" qui la raconte, ce qui met en jeu les figures genettiennes relatives à l'ordre, à la durée ou à la fréquence, autant que les effets de modalisation de l'information narrative liées à la voix et à la focalisation. 
Certains, dans le sillage de Ricœur (1983), se servent encore de cette notion pour définir, sur un plan fonctionnel cette fois-ci, la production d'une configuration qui permet comprendre l'histoire en s'appuyant, notamment, sur un point de vue rétrospectif permettant une saisie globale des événements. L'accent est alors mis sur le travail de l'auteur en vue de rendre l'histoire signifiante ou compréhensible pour le lecteur. On peut ajouter qu'à ce même niveau fonctionnel, il est possible d'associer l'intrigue à un effet pratiquement opposé: à savoir la production d'une tension, provisoire ou définitive, qui joue sur une réticence intentionnelle dans la textualisation des actions. Cette intrigue intrigante (la redondance de l'expression est ici significative) est celle qui donne aux lecteurs l'expérience la plus saillante de la temporalité, puisque la possibilité de saisir la structure globale des événements est longtemps différée, alors que des configurations incertaines et provisoires sont échafaudées par les lecteurs tout au long de leur progression dans le texte, ce qui transforme la fabula en un réseau instable de virtualités. Cette dernière définition apparaît aussi comme celle, étroite, à laquelle se réfèrent la plupart des locuteurs ordinaires lorsqu'ils se plaignent que les récits "sans intrigue" manquent de relief, qu'ils sont ennuyeux. C'est également dans cette définition que le nœud de l'intrigue peut être considéré comme un dispositif induisant une tension qui oriente de récit vers son éventuel dénouement.

Dans toutes ces définitions parallèles, l'intrigue est toujours associée à une séquence du récit, mais des variables différencient le niveau sur lequel cette séquence se situe et la fonction qu'elle remplit dans l'interaction discursive. Pour certains, elle se situe au niveau de l'histoire racontée, pour d'autres, elle structure le discours qui raconte cette histoire, alors que les approches rhétoriques - celles de Brooks (1984), de Phelan (1989) ou de Sternberg (1993) en particulier - considèrent qu'elle relève de l'interrelation entre ces deux niveaux et de leur fonction discursive. Par ailleurs, d'un point de vue fonctionnel, il faut encore déterminer si cette double séquence consiste à rendre l'action compréhensible (RICCEUR, 1983) ou si elle vise au contraire à nouer une tension en rendant l'action temporairement incompréhensible - c'est-à-dire saisissable comme une totalité inachevée, qui demande un effort interprétatif accru en vue de compenser cette lacune intentionnelle en esquissant des virtualités sur la trame de l'histoire. Sur ce dernier point, trop souvent négligé par les approches structuralistes, on peut partir du principe que les rapports spécifiques qui s'établissent entre la séquence événementielle et la séquence textuelle peuvent refléter deux attitudes communicatives pratiquement opposées: ils peuvent servir à intriguer le lecteur ou, à l'inverse, à l'informer.
De manière à éviter tout malentendu terminologique, je suggère pour ma part de réserver le terme intrigue pour désigner la dynamique du récit intrigant, en relation avec ce que certains scénaristes désignent comme la production d'un "arc narratif". Cette métaphore insiste sur le rôle central joué par la déclivité ou le relief du récit $^{3}$, c'est-à-dire par le nouement et le dénouement d'une tension dans l'expérience esthétique. On pourra désigner les autres aspects que nous venons d'évoquer par les termes: séquence événementielle, séquence textuelle et configuration:

1. Approches formelles:

1.1. La séquence événementielle (désignée également par les termes fabula ou histoire) renvoie à la trame de l'histoire dans ses dimensions chronologique et causale. On peut associer cette structure à une propriété immanente du texte, même s'il s'agit en réalité d'une reconstruction mentale du lecteur qui se stabilise lorsque le récit est achevé et qui finit ainsi par correspondre à l'histoire effectivement racontée ou à son résumé.

1.2. La séquence textuelle (désignée également par les termes sujet, récit ou discours) renvoie à l'ordre dans lequel les informations concernant l'histoire sont introduites dans le récit. En cela, il s'agit bien d'une structure objective du texte, d'une série d'instructions, et non d'une reconstruction mentale du lecteur.

2. Approches fonctionnelles:

2.1. La configuration est un dispositif textuel dont la fonction est d'inscrire les événements racontés dans une totalité intelligible et de leur conférer un sens, qui peut être causal, intentionnel, explicatif, illustratif, moral ou autre.

2.2. L'intrigue est un dispositif textuel dont la fonction est d'intriguer le lecteur. Elle se noue par l'établissement d'une tension et elle vise à orienter l'attention en direction d'un dénouement attendu. La tension narrative repose sur la mise en relation de l'événement tel que le lecteur peut se le représenter à un stade précoce de son développement, avec des virtualités passées, actuelles ou futures. Le récit intrigant (ou récit à intrigue) se caractérise par une forme minimale d'immersion du lecteur dans un monde possible distinct de son monde actuel et, par ailleurs, par la création d'un intérêt lié à cette projection dans une expérience simulée, qui se caractérise par un sentiment de suspense, de curiosité ou de surprise.

\footnotetext{
Voir aussi, dans le champ de la linguistique, l'approche de Bronckart (1985; 1996) qui s'inscrit dans le cadre d'un interactionnisme sociodiscursif et recourt à la même métaphore.
} 
Sur la base de cet inventaire, il devient possible de reposer la question de l'extension du corpus des récits qui comportent une intrigue, dans et hors de la littérature. Certes, par définition, tous les récits sont censés posséder, au moins sous une forme implicite, une double séquence: celle des événements racontés et celle de l'ordre de leur présentation, mais les fonctions narratives liées à la configuration d'un savoir ou à la mise en intrigue des événements prennent un poids différent suivant les genres envisagés. Soit, à l'instar de Meir Sternberg (1992), on définit la narrativité sur une base fonctionnelle en affirmant que cette dernière dépend de la production d'effets de suspense, de curiosité et de surprise, ce qui entraîne que seuls les récits intrigants seront considérés comme pleinement narratifs. Soit, et je pense que c'est une position plus nuancée, on considérera que la narrativité dépend avant tout, sur un plan formel, de la présence d'une double séquence, textuelle et événementielle, alors on considérera qu'il existe différentes formes de narrativité qui remplissent différentes fonctions, en relation avec des genres de discours spécifiques. On acceptera alors d'envisager l'existence de récits plus ou moins mimétiques, dans lesquels les jeux sur l'interséquentialité peuvent avoir pour objectif de configurer un savoir (pour les récits informatifs) ou d'attirer l'attention sur la matérialité discursive du texte (pour les romans modernistes antiimmersifs) et non le désir d'intriguer le lecteur et de l'immerger dans l'histoire 4 . Dès lors, l'absence de dispositifs intrigants au sein de certains récits n'apparaîtra pas comme un défaut, mais au contraire comme une qualité assurant le succès de l'acte de langage.

\section{Les phases de l'intrigue}

D'un point de vue séquentiel, l'intrigue qui rythme l'intrigue au-delà des limites de la phrase s'articule de la manière suivante: à une phase initiale de nouement succède généralement (mais pas nécessairement) une phase ultérieure de dénouement, qui vient clore le récit ou un épisode de celui-ci. Le dénouement répond ainsi aux incertitudes introduites par le nœud, qui apparaît pour sa part comme un inducteur d'incertitude encourageant la participation active du lecteur à l'élaboration du sens du texte ${ }^{5}$. Il faut ajouter que cette structure séquentielle ne condamne nullement le récit à se conformer au standard hollywoodien du scénario en trois actes, lui-même inspiré des standards dramatiques préconisés par Aristote ou par la fameuse "pyramide" de Gustav Freytag (1863).

\footnotetext{
4 Sur cette distinction, voir Baroni (2009).

5 Ce questionnement peut parfois prendre une forme explicite. C'est notamment le cas dans les feuilletons qui soulignent les incertitudes de l'intrigue, soit pour renforcer l'intérêt du lecteur pour la suite de l'histoire, soit pour faciliter sa compréhension des enjeux narratifs, comme c'est souvent le cas dans les bandes dessinées destinées aux enfants.
}

Un simple chapitre de roman peut présenter plusieurs intrigues parallèles, dont certaines peuvent se nouer ou se dénouer, tandis que d'autres peuvent être laissées en l'état d'ouverture, de sorte que la fonction de la scansion chapitrale, ainsi qu'on le verra plus loin, peut servir à la fois de nœud, de péripétie ou de dénouement. Pour définir la manière dont se noue le récit, il s'agit cependant de distinguer clairement:

1. ce qui relève d'une textualisation réticente de la séquence événementielle, qui induit une indétermination intentionnelle concernant le passé, le présent ou le futur de l'action racontée;

2. ce qui touche aux efforts cognitifs du lecteur visant à réagir à cette indétermination en formulant des pronostics ou des diagnostics concernant l'action racontée, ce qui lui permet de construire une représentation mentale de la fabula sous la forme d'une configuration incertaine, précaire et en constante évolution;

3. ce qui relève enfin d'un effet esthétique résultant de la mise en intrigue, qui prend la forme du suspense ou de la curiosité$^{6}$, deux modalités alternatives de la tension narrative que l'on peut corréler au type de traitement cognitif (pronostic ou diagnostic) et à l'incertitude engendrée par la textualisation de l'histoire.

Il est important de noter que même si certaines stratégies textuelles engendrent plutôt du suspense et d'autres plutôt de la curiosité, il peut arriver qu'une même situation puisse être interprétée comme relevant des deux effets en même temps. Par exemple, dans une enquête, le lecteur peut être amené à produire un diagnostic concernant l'identité du coupable éventuel (qui a tué X?) tout en faisant des pronostics concernant les chances de succès de l'enquêteur (va-t-il élucider le meurtre?). En fin de compte, l'identification de la résultante affective (suspense ou curiosité) dépendra alors de la nature de l'activité cognitive du lecteur, et si les deux effets sont différenciés sur un plan conceptuel, ils ne sont pas nécessairement incompatibles et peuvent, dans certains cas, devenir pratiquement indiscernables dans une configuration textuelle déterminée.

Entre nœud et dénouement est intercalée une phase d'attente caractérisée par une réticence à livrer des informations qui pourraient dénouer la tension initiale. Cette phase peut être associée au sens étymologique de

\footnotetext{
6 Je renonce à l'usage du terme "fonction thymique", utilisé dans $L a$ Tension narrative (2007), de manière à ne pas compliquer inutilement la terminologie, tendance qui a été parfois reprochée aux narratologues et qui a atteint son paroxysme dans la sémiotique greimasienne. Je ne mentionne pas davantage la surprise à cette étape de l'analyse, dans la mesure où, contrairement au suspense et à la curiosité, cet effet ponctuel ne permet pas de structurer un arc narratif alternant tension et résolution.
} 
la péripétie, c'est-à-dire ce qui tourne autour du pot, ce qui advient sans que cela conduise directement au but. La fonction discursive de ces péripéties est alors d'entretenir ou de moduler la tension introduite par le nœud. Dans le cas des intrigues les plus planifiées, ces détours pourront ultérieurement apparaître comme une suite logique de causes et d'effets qui, en dépit de leur caractère louvoyant, constituaient le meilleur chemin pour sortir du labyrinthe narratif, étant entendu que la voie la meilleure n'est pas forcément la plus directe, puisqu'il s'agit de jouir du voyage et non d'arriver à destination.

Le dénouement, quant à lui, est optionnel et ne constitue que l'une des virtualités de l'histoire. Ici encore, il s'agit de prendre le terme dans son sens fonctionnel, comme une réponse à un questionnement induit par une phase antérieure du discours. Il est ainsi possible d'opposer la clôture du texte, qui correspond formellement à l'interruption provisoire ou définitive du récit (ce dernier n'étant pas infini), et le dénouement de l'intrigue, qui correspond fonctionnellement à la résolution de la tension.

Ce modèle n'est donc pas incompatible avec ce qu'Umberto Eco (1979) appelait les "œuvres ouvertes" ou avec des récits sérialisés et plus ou moins improvisés. Certains feuilletons démontrent à l'envi que des intrigues non planifiées peuvent se prolonger indéfiniment et qu'en dépit de ce caractère émergent, une tension très efficace peut être entretenue, de rebondissement en rebondissement. Lorsqu'un épisode se clôt sur une situation tendue, la plupart des lecteurs jugent que l'intrigue se présente sous l'un de ses aspects les plus saillants, et le fait que, dans de nombreux cas, les auteurs ignorent la manière dont ils vont résoudre cette situation, ne paraît nullement de nature à remettre en cause un tel jugement. Jean-Cristophe Menu, en tant qu'auteur de bande dessinée, a décrit le plaisir singulier que l'on peut tirer de la discontinuité temporelle induite par le feuilleton, tout en soulignant que le caractère improvisé de l'écriture peut participer à ce charme:

A posteriori, je crois que ce principe de discontinuité temporelle a été primordial dans mon attachement premier à la bande dessinée. Le journal de Spirou se présentait comme une collection de feuilletons: deux pages d'un récit, la production d'un suspense en fin de deuxième page, et la mention (à suivre). Dans l'attente du numéro de la semaine suivante, l'imagination était en effervescence, l'histoire se poursuivait à travers de multiples hypothèses dans l'imaginaire, bref cet agencement de récits à suivre induisait un processus de désir. [...] Lorsque le feuilleton est combiné à l'improvisation, le plaisir de l'auteur rejoint celui du lecteur. La suspension du récit dans l'imaginaire, effectuée par le lecteur, est également vécue par l'auteur, qui dans le même laps de temps, ignore lui aussi ce qui va se passer. Il s'ensuit une véritable communion entre l'auteur et le lecteur, basée sur la surprise, dans un interstice de temps imaginaire partagé (MENU, 2010, p. 303-306).

Il faut ajouter que les intrigues émergentes ne sont pas propres uniquement à des récits associés à une production industrielle, fondée sur la sérialisation et impliquant une forme de bricolage narratif7. Marc Escola a montré que le principe de "causalité régressive", qui fonde la plupart des modèles narratologiques de la période structuraliste, est inadéquat pour décrire les "modes de composition privilégiés par les romanciers des XVII et XVIII" siècles" (2010, n.p.). En effet, le roman-fleuve se présente davantage comme une errance imprévisible que comme une architecture romanesque minutieusement organisée à partir son point final. Ces exemples montrent que la dynamique de l'intrigue n'est pas toujours liée à une planification, en revanche, elle dépend de manière essentielle des dispositifs dont la fonction est de produire une tension susceptible d'emporter le lecteur dans sa progression textuelle, comme le courant d'un fleuve. S'il y a théoriquement autant d'arcs narratifs qu'il y a de questions laissées en suspens dans le récit, on peut cependant considérer que la tension globale constitue un arc majeur, comme un faisceau formé par l'addition de tous les arcs individuels formant la "trame" de l'intrigue.

Il s'agit maintenant de préciser les rapports qui existent entre les deux modalités du nœud de l'intrigue et la sémantique de l'action. Cette explication sera volontairement concise, car cette dimension de l'analyse a déjà été largement développée dans mes ouvrages précédents (notamment dans La Tension narrative). Il ne s'agira de donner qu'un aperçu superficiel des principaux critères sémantiques qui permettent de qualifier la tension de narrative. Cette tension est spécifiquement liée à la représentation d'une histoire. Il existe en effet d'autres formes de tension esthétiques: par exemple, dans la musique instrumentale, une tension peut être modulée par un jeu de consonance-dissonnance à l'intérieur du système tonal, ou par une variation du volume, du timbre ou du rythme, jusqu'à atteindre un climax et une forme de résolution. Les discours argumentatifs peuvent également être marqués par une progression culminant dans une synthèse conclusive. Bref, tout phénomène esthétique ou discursif, pour autant qu'il s'inscrive dans une temporalité et dans une séquentialité, peut potentiellement être décrit comme s'organisant séquentiellement par le biais d'une alternance tension-détente. En revanche, ce qui fait la

\footnotetext{
Pour une réévaluation de la qualité esthétique de l'écriture improvisée des séries télévisées, je renvoie à mon article "Intrigue et personnages dans les séries évolutives: quand l'improvisation devient une vertu" (BARONI, 2016).
} 
spécificité de la tension que l'on associe à une mise en intrigue, c'est qu'elle vise à produire chez le lecteur la représentation mentale dynamique d'une histoire. Prise dans ce sens étroit, la tension narrative engendrée par la mise en intrigue, apparaît bien comme un phénomène spécifique aux récits mimétiques, en dépit du fait que l'alternance tension-détente constitue une expérience beaucoup plus primitive et générale de la temporalité.

\subsection{Mise en intrigue par la curiosité}

Pour décrire le fonctionnement de la mise en intrigue par la curiosité, il est nécessaire de définir le ou les éléments de la sémantique de l'action qui sont occultés par le discours: souvent, l'irruption d'un nouveau personnage suscite des interrogations, ses intentions peuvent apparaître énigmatiques, il peut arriver également que nous nous interrogions sur son identité ou ses motivations. Même le lieu ou le temps d'une scène peuvent faire l'objet d'une explicitation retardée. Bertrand Gervais a tenté de dresser un inventaire exhaustif du réseau conceptuel de l'action qui peut se révéler utile lorsqu'il s'agit de définir l'élément sur lequel porte le déficit d'information:

Le schème interactif est cette structure, cet ensemble de catégories organisées entre elles permettant l'identification de l'action, quel que soit son type de manifestation: dans un récit ou dans le monde. Le schème interactif préexiste à la lecture et il trouve ses racines dans la connaissance pratique du domaine de l'action et de sa conceptualisation par le langage ordinaire; il vaut donc comme modèle de la configuration générale de la pré-compréhension pratique de l'action, pré-compréhension posée comme garantie de la capacité du lecteur de comprendre adéquatement la représentation d'une action (GERVAIS, 1990, p. 80).

Sans entrer dans le détail des schématisations de l'action que propose Gervais, je me contenterai de souligner que, de manière idéale, le lecteur d'un récit dont l'action est représentée avec clarté et complétude devrait être en mesure de répondre aux questions suivantes:

- Cadre: où et quand l'action se déroule-t-elle?

- Agent: qui agit? quel est le rôle du personnage dans l'histoire? quelles sont ses relations avec les autres personnages?

- Opération: que fait l'agent? quelle est son intention? quel est le but visé par son action? quel sont les mobiles ou les motifs qui le poussent à agir?

- Accessoires: quels sont les moyens mis en œuvre? quels sont les accessoires requis ou compatibles pour la réalisation de l'action?
Naturellement, tous ces éléments n'ont pas à être systématiquement décrits par un récit coopératif, mais lorsqu'ils sont laissés en blanc, c'est que le narrateur suppose qu'ils sont implicites ou insignifiants dans un contexte donné, et que l'interprète n'aurait donc aucun mal à les compléter (plus ou moins librement) en se fondant sur ses compétences encyclopédiques. La mise en intrigue par la curiosité suppose au contraire qu'au moins un de ces éléments apparaisse volontairement occulté, de manière à ce que le lecteur soit obligé de formuler un diagnostic à partir d'indices indirects. Par "volontairement occulté", il faut comprendre que la dissimulation d'un élément pertinent du réseau conceptuel de l'action est saillante (et non pas discrète) et que, par conséquent, le lecteur est conduit à l'interpréter comme une stratégie intentionnelle de l'auteur.

Lorsque le récit intrigue le lecteur par le biais de la curiosité, le narrateur renonce ouvertement à la transparence de la représentation, et la matérialité de son discours tend souvent à devenir plus visible. Par conséquent, cette modalité de la mise en intrigue peut s'accommoder d'une immersion affaiblie et elle permet souvent de retenir l'intérêt du lecteur dans les incipits romanesques ou dans les débuts de chapitre, qui sont marqués par une rupture narrative, par un changement de temps, de lieu ou de focalisation. Il faut cependant ajouter que la curiosité peut aussi fonctionner en régime d'immersion maximale lorsqu'elle est intégrée au monde diégétique, par exemple lorsque le lecteur partage la curiosité d'un protagoniste confronté à une énigme ou aux intentions obscures d'un autre personnage.

\subsection{Mise en intrigue par le suspense}

Le suspense est créé lorsque le développement, l'issue ou les conséquences d'un événement demeurent incertains, mais néanmoins partiellement prévisibles. L'interprète est alors encouragé à produire un pronostic sur le développement ultérieur de la séquence événementielle. La réticence textuelle se manifeste dans ce cas par un respect au moins partiel de la chronologie des événements, ce qui a pour effet de retarder l'exposition d'informations concernant un futur que le lecteur souhaiterait connaître d'emblée. D'une manière générale, sur le plan de la logique actionnelle, le suspense se noue lorsqu'un événement habituel ou routinier se trouve bouleversé. Par conséquent, cette rupture suppose que les "scripts" 8 " régissant les actions prévisibles de la vie quotidienne soient connus du lecteur, ou du moins, si l'action se déroule dans un monde éloigné du nôtre (science-fiction, roman historique, etc.) que ces séquences

\footnotetext{
8 Cette notion de "script" voir (BARONI, 2007, p. 167-179).
} 
d'actions prévisibles soient décrites de manière minimale. Diverses formes de situations instables peuvent naître de la transgression d'un script, les plus fréquentes étant:

- les buts difficiles à atteindre qui nous projettent vers leur accomplissement incertain;

- les relations polémiques qui dessinent les contours d'un conflit devant déboucher sur une issue indécise;

- les événements catastrophiques qui menacent le protagoniste.

On peut ajouter à cet inventaire quelques matrices interactives fondamentales ${ }^{9}$, dont le développement peut déboucher sur un très vaste répertoire de virtualités:

- les contrats honnêtes ou trompeurs, qui peuvent être réalisés ou transgressés;

- les dons positifs ou négatifs, qui peuvent être suivis de contre-dons, eux-mêmes positifs ou négatifs;

- les ordres ou les interdictions, qui peuvent être respectés ou transgressés;

- les méfaits ou les transgressions, qui ouvrent sur la virtualité d'une sanction.

À la suite des travaux de Claude Bremond, on considère en général que ces processus événementiels instables, qui fournissent l'armature à de nombreuses intrigues, se déploient selon une logique en triade comprenant des étapes marquées par le déploiement de virtualités actionnelles.

Éventualité $\rightarrow$ Passage à l'acte $\rightarrow$ Achèvement $\rightarrow$ Non-passage à l'acte $\rightarrow$ Inachèvement

(BREMOND, 1973, p. 131).

Le caractère binaire des virtualités identifiées par Claude Bremond apparaît cependant réducteur au regard du vaste réseau de virtualités qui peut se déployer à partir d'une situation interactive donnée. Même une formalisation grossière des interactions les plus générales (contrats, dons, ordres, interdictions, méfaits, etc.) met en évidence l'existence de virtualités beaucoup plus nombreuses à chaque étape de leur développement, puisqu'il faut également définir les modalités du passage à l'acte et les conséquences pratiques de cette modalité: par exemple le passage à l'acte de la transgression d'un contrat implique la virtualité d'une punition qui, ellemême, dépend de la découverte du méfait et de la manière dont ce méfait sera jugé, ce qui implique de nombreuses combinaisons possibles. On peut donc schématiser les étapes plus ou moins prévisibles d'une interaction, mais on peut beaucoup plus difficilement prévoir la forme concrète de son actualisation, qui laisse beaucoup de place à l'incertitude, et permet de ménager du suspense.

\footnotetext{
9 Sur les matrices interactives, voir (BARONI, 2007: 179-198)
}

Il faut encore préciser que le suspense dépend de manière fondamentale de l'immersion du lecteur dans l'histoire racontée, de sorte que, même si le développement de l'intrigue apparaît comme relativement prévisible (soit parce qu'il est stéréotypé, soit parce que le récit a déjà été lu ou "spoilé"), il conserve une partie de son intérêt. En effet, dans une scène de suspense, le lecteur est amené à partager la perspective temporelle des personnages, ce qui entraîne que les événements conservent leur ouverture vers un avenir potentiel. Ainsi que l'affirme Marie-Laure Ryan, l'immersion permet de rendre la scène présente dans l'esprit du lecteur, à tel point que:

il importe peu de savoir si la situation est vraie ou fausse, ou si son développement est connu ou inconnu, étant donné que la simulation la rend temporairement vraie et présente, et du point de vue du présent, le futur n'a pas encore eu lieu (RYAN, 2001, p. 156, m.t.).

Le suspense exige ainsi que la qualité immersive du texte soit suffisante pour qu'une projection dans l'espacetemps de la simulation soit possible, ce qui exige une caractérisation minimale des lieux, des personnages et des circonstances de l'action. Il faut toutefois préciser que cette immersion dans le monde raconté ne nécessite pas nécessairement de s'identifier ou de partager intégralement la perspective cognitive du protagoniste, dans la mesure où un suspense efficace repose souvent sur l'anticipation par le lecteur d'un danger que le personnage ignore. Dans un tel cas, l'immersion consiste à se situer imaginairement dans le même monde que le personnage, tout en demeurant un témoin impuissant et muet, qui ne peut assister que passivement aux événements qui s'y déroulent. On reconnaît ici l'ambivalence de la simulation narrative: un pied dans le monde raconté nous place dans la perspective d'un drame imminent, qui nous donne envie de sauver les personnages menacés, un pied dans le monde réel nous condamne au rôle tragique de Cassandre, dont les avertissements ne peuvent être entendus de personne au sein de la fiction.

\subsection{Surprise}

À côté de la curiosité et du suspense, qui sont les deux modalités de la tension narrative qui structurent l'intrigue, il faut encore mentionner la surprise, qui est ponctuelle, mais extrêmement importante du point de vue cognitif, car elle permet de souligner rétrospectivement le caractère sous-déterminé des pronostics ou des diagnostics qui ont guidé la progression du lecteur dans le texte. La fonction de la surprise est généralement de nous faire prendre conscience des codes sur lesquels s'érige notre interprétation du monde raconté: le décalage entre nos attentes et ce que nous lisons effectivement dans le 
récit permet de renouveler et d'actualiser un répertoire de stéréotypes ${ }^{10}$, qui nous servent aussi bien à comprendre et à anticiper le développement des textes que lisons qu'à participer adéquatement aux événements réels auxquelles nous sommes confrontés. Par exemple l'intrusion du hasard dans l'explication finale livrée par le dénouement d'un récit policier ou le décès d'un enquêteur aveuglé par ses hypothèses alambiquées qui s'opposent aux lois probabilistes auxquelles se soumet l'intrigue, peuvent nous amener à prendre conscience des codes régissant un genre littéraire, tout en offrant une leçon existentielle sur notre aveuglement face à un monde privé de fondements métaphysiques ${ }^{11}$. Il faut aussi noter que le nœud, en perturbant un ordre établi dont découle l'aventure, se signale souvent par une surprise plus ou moins marquée.

\section{La caractérisation des personnages: entre indétermination et surdétermination}

De manière à illustrer comment une analyse formelle du récit peut rejoindre une analyse fonctionnelle de l'intrigue, je me focaliserai sur une analyse des personnages, dont on verra qu'au-delà de leur caractérisation thématique, se pose également la question de la manière dont les informations les concernant sont introduites dans le cours du récit et déterminent la tension narrative. Dans ses travaux, Vincent Jouve insiste sur la nécessité de passer d'une description formelle du personnage à une analyse de son effet dans la dynamique de la lecture:

Les figures construites par le texte ne prennent sens qu'à travers la lecture. Le sujet lisant est, en dernière instance, celui qui donne vie à l'œuvre. [...] Une description formelle, voire fonctionnelle, du personnage n'est plus suffisante. À la question de savoir ce qu'est un personnage doit succéder cette autre: qu'advient-il de lui dans la lecture? Ou encore: comment et à quelle(s) fin(s) le lecteur l'appréhende-til? (JOUVE, 1992, p. 107).

Si l'on considère l'inventaire des rôles actanciels proposé par Greimas (qui comprend les fonctions de sujet, objet, adjuvant, opposant, destinateur, destinataire), il devient par exemple nécessaire de tenir compte de la sous-détermination provisoire de ces rôles durant le cours de la lecture. Sur ce point, Umberto Eco a d'ailleurs souligné la difficulté de lier une structure actancielle aux nœuds textuels tels qu'ils se présentent au lecteur qui progresse dans le récit:

\footnotetext{
$\overline{10 \text { Voir Dufays (2010). }}$

11 J'ai illustré ce cas en m'appuyant sur un conte célèbre de Borges intitulé La Mort et la boussole (BARONI, 2007, p. 371-385).
}

La construction de la charpente profonde est le résultat final d'une inspection critique et [...], comme telle, elle ne survient qu'à une phase avancée (et réitérée) de lecture. Mais dans notre optique présente (on essaie de cerner les nœuds textuels où un type déterminé de coopération est requis), la décision théorique devient désespérée. Nous savons, du moins quand la reconstruction critique est effectuée, qu'un texte a ou devrait avoir telle structure actancielle, mais nous pourrions difficilement dire à quelle phase de la coopération le Lecteur Modèle est invité à l'identifier (ECO, 1985, p. 228-229).

À nouveau, les schémas hérités des modèles structuralistes peuvent se révéler utiles pour l'analyse de la tension narrative, mais ils doivent être temporalisés et relativisés, c'est-à-dire interprétés comme des virtualités mobiles qui s'échafaudent dans le cours de la lecture. La schématisation construite par le lecteur à une étape donnée du récit se présente comme une structure possible qui contribue à la dynamique du récit et cette potentialité peut être reconfigurée à un stade ultérieur de la progression dans le texte. C'est ainsi que certains personnages qui semblent à première vue agir comme des adjuvants, peuvent se révéler, plus tard, être des opposants (ce qui engendre une surprise) ou que des êtres aux rôles ambigus peuvent susciter notre curiosité aussi longtemps que leur fonction dans l'intrigue n'a pas été clarifiée. Les récits longs et complexes démontrent également la mutabilité des rôles: alors que tel personnage peut occuper le centre de l'intrigue avant de passer au second plan, d'autres peuvent changer de camp, évoluer, nouer des alliances avant de les rompre à un stade ultérieur du récit. Ce sont donc l'indétermination et/ou l'évolution des rôles sur lesquelles il s'agit de mettre l'accent, plutôt que la détermination pour chaque personnage d'une fonction unique dans une structure globale, de sorte qu'il devient possible d'établir un lien entre cette mobilité de la caractérisation des personnages et la dynamique de l'intrigue.

Par ailleurs, il est évident que l'intensité du suspense dépend en grande partie de l'investissement affectif du lecteur envers le sort des personnages. Ainsi que le souligne Françoise Lavocat, les "théories contemporaines traitant de l'identification et de l'empathie" sont allées jusqu'à contester "la différence entre personne et personnages" (2016, p.347). Dans une approche rhétorique, James Phelan définit quant à lui la "fonction mimétique du personnage" comme l'art de "créer l'illusion d'une personne plausible" (PHELAN, 1989, p.11) et il montre que cette épaisseur psychologique joue un rôle essentiel dans l'impact émotionnel de ses actions sur le public et dans l'évolution de la tension du récit. Du point de vue de la lecture, Jouve affirme lui aussi que l'investissement pulsionnel du lecteur - c'est-à-dire la 
possibilité "de vivre par procuration un certain nombre de situations fantasmatiques" (JOUVE, 1992, p. 110) - dépend de la caractérisation plus ou moins réaliste et détaillée des agents:

Le personnage sera ainsi à étudier comme élément du sens (fonction narrative et indice herméneutique), illusion de personne (objet de la sympathie ou de l'antipathie du lecteur) et alibi fantasmatique (support d'investissements inconscients) (JOUVE, 1992, p. 111).

Il faut cependant ajouter, ainsi que le soulignait Hitchcock, que la sympathie n'est pas un ingrédient absolument nécessaire à l'investissement pulsionnel du lecteur dont dépend la production du suspense:

\begin{abstract}
Prenons un [...] exemple, celui d'une personne curieuse qui pénètre dans la chambre de quelqu'un d'autre et qui fouille dans les tiroirs. Vous montrez le propriétaire de la chambre qui monte l'escalier. Puis vous remontez sur la personne qui fouille et le public a envie de lui dire: 'Faites attention, faites attention, quelqu'un monte l'escalier'. Donc, une personne qui fouille n'a pas besoin d'être un personnage sympathique, le public aura toujours de l'appréhension en sa faveur. Évidemment, si la personne qui fouille est un personnage sympathique, alors vous doublez l'émotion du spectateur, par exemple avec Grace Kelly dans Rear Window (TRUFFAUT, 1975, p. 80).
\end{abstract}

Cet exemple souligne l'importance de ne pas simplifier à outrance l'analyse fonctionnelle des dispositifs narratifs, les processus d'investissement affectif du récepteur oscillant entre la proximité maximale de l'identification ou de l'empathie et d'autres rapports plus distancés. Des travaux récents sur les neurones miroirs nous ont quant à eux rendus attentifs à la capacité des récepteurs d'un récit de se projeter dans le monde narratif, par un phénomène de simulation mentale relativement autonome des jugements moraux sur lesquels se fondent la sympathie ou l'antipathie. L'exemple de Hitchcock suggère cependant que l'engagement émotionnel du spectateur peut se fonder sur un excès de savoir par rapport au personnage menacé, ce qui souligne plutôt l'asymétrie cognitive entre ces deux instances. En l'occurrence, le spectateur "voit" le propriétaire monter les escaliers, de sorte l'empathie, si elle opère, ne repose pas sur une identification totale avec le plan cognitif du personnage, qui ignore cette information, mais sur un investissement d'un autre ordre. Malgré tout, l'angoisse de la sanction qui accompagne la réalisation d'un acte transgressif pourra être activée, alors que la posture cognitive, en revanche, correspondra plutôt à celle du témoin ${ }^{12}$ d'un drame ou d'un complice confronté à son impuissance à infléchir le cours des événements, l'oubli partiel du caractère artificiel de la représentation accentuant cet effet.

Quoi qu'il en soit, même si l'investissement affectif du lecteur est complexe, nous voyons que la construction textuelle du personnage joue un rôle essentiel pour la dynamique de l'intrigue: d'un côté, la relative indétermination de sa fonction dans le récit peut contribuer à nourrir un sentiment de curiosité plus ou moins durable, d'un autre côté, le personnage doit posséder une certaine épaisseur pour que les aléas de son destin puissent intéresser le lecteur et entretenir un sentiment de suspense ${ }^{13}$, ce qui passe généralement par une forme de surdétermination que Barthes associait à l'"effet de réel" (1968). Il faut encore préciser que cet effet de réel ne doit pas être confondu ici avec le réalisme de la fiction. Un personnage appartenant à un monde fantastique ou science-fictionnel, comme Paul Atréïde dans le Cycle de Dune (qui finit par devenir un vers des sables) ou Frodon Saquet dans Le Seigneur des Anneaux, peuvent acquérir, au fil du récit et en dépit de leur caractère irréaliste, une épaisseur psychologique considérable. Françoise Lavocat caonstate d'ailleurs:

Il est [...] probable que l'empathie s'exerce plus librement à l'égard des personnages fictionnels, justement parce qu'elle ne nous invite pas à l'action. Keen suppose même que, contrairement à ce qui est couramment admis par la critique littéraire, les fictions irréalistes touchent davanatge les lecteurs ou les spectateurs, car la réponse empathique est favorisée par la distance avec le réel. La fictionnalité exhibée aurait ainsi un rôle protecteur, garantissant au lecteur la possibilité de développer une réponse empathique sans risque ni engagement de sa part (2006, p.220) (LAVOCAT, 2016, p. 362)

En résumé, lorsque le rôle joué par un personnage manque de clarté, lorsque que ses intentions demeurent obscures u que nous le soupçonnons de conserver un secret, ce dernier agit comme un inducteur de curiosité, tandis que le suspense se nourrit de l'accumulation d'informations qui lui permettent d'acquérir de la profondeur, de lui conférer une consistence ontologique de sorte qu'il devient possible de s'attacher à cette personne fictive, ce qui n'implique pas nécessairement que celle-ci doive se conformer à des critères strictement

\footnotetext{
${ }^{12}$ Cette posture de témoin correspond à l'une des trois postures fondamentales que Monika Fludernik (1996), dans le prolongement du "cercle" de Stanzel (voir Patron, 2009, p. 79-97), associe à l'expériencialité du récit mimétique.

13 On pourrait aussi rattacher à cette double dynamique les réflexions de Philippe Hamon (1983) sur la fonctionnalité et la qualification du personnage.
} 
réalistes. À ces deux aspects s'ajoute le caractère instable sur le long terme des rôles tenus par les personnages, qui peut autant servir à renforcer le suspense ou la curiosité du récit. Bref, la tension narrative se nourrit à la fois de la sous-détermination intrigante et de la surdétermination attachante des personnages, ce qui explique pourquoi, dans de nombreux romans, la curiosité domine dans les premières pages avant de faire place, à mesure que l'on progresse dans le récit et que les enjeux et les rôles de chacun se précisent, à la dynamique du suspense ${ }^{14}$. Les deux effets ne sont cependant pas incompatibles: il arrive que des personnages surdéterminés possèdent malgré tout des secrets, produisant ainsi une indétermination locale tout en préservant leur épaisseur existentielle. Quoi qu'il en soit, l'intensité de l'intrigue n'est pas seulement proportionnelle aux enjeux événementiels, mais également à la pression cumulée de tous les événements antérieurs qui ont contribué à nous intéresser au vécu d'une personne dont le statut ontologique est solidement ancré dans le monde raconté.

\section{Conclusion}

L'une des principales innovations apportées par la narratologie postclassique pour l'analyse des œuvres littéraire est d'éclairer la fonction de l'intrigue en la resituant au cour de l'interaction entre le texte et le lecteur. Les liens entre l'intrigue et l'histoire racontée doivent ainsi être redéfinis sur une base rhétorique, en insistant sur les dispositifs textuels qui visent à dynamiser la progression du lecteur dans le récit, notamment à travers l'articulation de virtualités qui se greffent sur la trame des événements racontés. L'histoire susceptible d'intriguer le lecteur est ainsi appréhendée comme une construction mentale précaire, une forme en mouvement qui ne cesse de se reconfigurer tout au long de l'avancée dans le texte. Il s'agit véritablement, pour le lecteur, d'interpréter le récit, comme un musicien interprète la partition d'un compositeur. Seule une performance imaginative permet de donner vie à l'œuvre, et sans cette participation active du lecteur, le récit n'est qu'une suite d'instructions imprimées sur une feuille de papier. Dans une perspective cognitiviste, David Herman (2002, p. 14) est allé jusqu'à suggérer de remplacer le substantif histoire (story) par le groupe nominal monde de l'histoire (storyworld), de manière à indiquer l'importance des virtualités envisagées par le lecteur à différentes étapes de sa progression dans le texte:

le monde de l'histoire [storyworld] souligne la façon dont les interprètes du récit reconstruisent une séquence d'états, d'événements et d'actions, non

\footnotetext{
$\overline{{ }^{14} \text { Sur cette alternance, }}$ je renvoie à Gervais (1990).
}

seulement de manière additive ou incrémentielle, mais intégrative ou "écologique"; les récepteurs ne tentent pas seulement de reconstituer des fragments de l'action dans un montage linéaire, mais ils tentent de surcroît de mesurer l'importance de la chronologie qui se dégage vis-à-vis des alternatives possibles qui pourraient se déployer dans le monde au sein duquel les événements racontés se sont déroulés (HERMAN, 2002, p. 14, m.t.).

La prise en compte du rôle central joué par les virtualités de l'histoire, qui constituent l'énergie potentielle du récit, conduit à redéfinir l'intrigue non plus comme un simple synonyme de séquence événementielle (fabula), mais comme une "matrice de possibilités ontologiquement instable" (DANNENBERG, 2008, p. 13). Or, ce réseau évolutif des histoires virtuelles qui s'articulent sur le fil de l'histoire actuelle, doit autant son existence au texte proprement dit, à sa matérialité discursive, qu'à l'imagination du lecteur, qui comble des lacunes intentionnelles du récit et qui compense sa réticence en esquissant des scénarios possibles, ce qui déplace l'analyse de la description des structures textuelles vers l'étude des dispositifs intrigants et de leurs effets. Pour mettre en lumière ces mécanismes textuels dont dépend l'énergie cinétique de la lecture, il est alors nécessaire de fusionner des approches traditionnellement disjointes: d'une part, l'analyse fonctionnelle des dispositifs narratifs, et d'autre part, la description des formes textuelles à travers lesquelles ces fonctions s'incarnent dans le contexte d'une œuvre singulière.

De nombreux éléments peuvent se combiner pour nouer une intrigue: il ne s'agit pas uniquement (ou même nécessairement), à un stade qu'en rhétorique on appellerait l'inventio, d'élaborer une trame d'événements mystérieux et/ou conflictuels qui formeront la fabula. La capacité de cette histoire à intriguer le lecteur dépend aussi étroitement de la dispositio et de l'elocutio, c'està-dire des effets de voix ou de point de vue, d'ordre, de durée, de fréquence, des temps verbaux qui expriment les procès, du découpage chapitral, de l'usage des pronoms ou des adverbes temporels, des isotopies lexicales ou des allusions intertextuelles qui orientent l'interprétation dans une direction déterminée. La manière de caractériser le personnage, ainsi qu'on l'a vu, détermine aussi directement le profil tensif de l'intrigue, soit en jouant sur une surdétermination attachante, soit en introduisant une sous-détermination intrigante de ses intentions ou de ses rôles. Bref, cette approche fonctionnelle suggère d'adopter vis-à-vis du récit une démarche intégrative, en faisant converger de nombreuses perspectives qui ont été trop souvent disjointes, tout en replaçant la dynamique de l'intrigue au cœur de l'analyse. Je suis personnellement convaincu que l'immersion du lecteur dans un monde 
raconté, ainsi que le nouement et le dénouement d'une tension au cœur de cette expérience simulée, constituent, sur un plan anthropologique, la raison d'être des récits mimétiques, même si ces derniers ont pu, au cours de l'histoire de la littérature, remplir d'autres rôles.

\section{Références}

ABBOTT, H. Porter. Story, Plot, and Narration. In: HERMAN, David (Org.). The Cambridge Companion to Narrative. Cambridg: Cambridge University Press, 2007. p. 39-51.

BARONI, Raphaël. Intrigue et personnages dans les séries évolutives: quand l'improvisation devient une vertu. Télévision, n. 7, p. 31-48, 2016.

BARONI, Raphaël. L'Euvre du temps. Poétique de la discordance narrative. Paris: Seuil, 2009.

BARONI, Raphaël. La Tension narrative. Suspense, curiosité et surprise. Paris: Seuil, 2007.

BARONI, Raphaël; REVAZ, Françoise (Org.). Narrative Sequence in Contemporary Narratology. Columbus: Ohio State University Press, 2016.

BARTHES, Roland. L'effet de réel. In: Le Bruissement de la langue. Paris: Seuil, 1984 (1968). p. 179-187.

BREMOND, Claude. Logique du récit. Paris: Seuil, 1973.

BRONCKART, Jean-Paul. Activité langagière, textes et discours. Paris: Delachaux \& Niestlé, 1996.

BRONCKART, Jean-Paul. Le Fonctionnement des discours. Paris: Delachaux et Niestlé, 1985.

BROOKS, Peter. Reading for the Plot. Design and Intention in Narrative. Cambridge and London: Harvard University Press, 1992 (1984).

DANNENBERG, Hilary. Coincidence and Counterfactuality. Plotting Time and Space in Narrative Fiction. Lincoln and London: University of Nebraska Press, 2008.

DANNENBERG, Hilary. Plot. In: Routledge Encyclopedia of Narrative Theory. London: Routledge, 2005. p. 435.

DUFAYS, Jean-Louis. Stéréotype et lecture. Essai sur la réception littéraire. Bruxelles: Peter Lang, 2010 (1994).

ECO Umberto. Lector in Fabula. Paris: Grasset, 1985.

ECO, Umberto. L'œuvre ouverte. Paris: Seuil, 1979.

ESCOLA, Marc. Le clou de Tchekhov. 2010. Retours sur le principe de causalité régressive, Fabula, Atelier de théorie littéraire. Disponível em: <www.fabula.org/atelier.
php?Principe_de_causalite_regressive\#_ftnref $21>$. Acesso em: 29 jul. 2014.

FLUDERNIK, Monika. Towards a 'Natural' Narratology. London: Routledge, 1996.

FREYTAG, Gustav. Die Technik des Drama. Leipzig: S. Hirtzel, 1863. Disponível em: <https://books.google.de/ books?id=CDYLAAAAIAAJ\&hl=fr $>$.

GERVAIS, Bertrand. Récits et actions: pour une théorie de la lecture. Longueuil: Le Préambule, 1990.

HAMON, Philippe. Le personnel du roman. Le système des personnages dans les Rougon-Macquart d'Émile Zola. Genève: Droz, 1983.

HERMAN, David Story Logic: Problems and Possibilities of Narrative. Lincoln: University of Nebraska Press, 2002.

JOUVE, Vincent. Pour une analyse de l'effet-personnage. Littérature, n. 85, p. 103-111, 1992.

KUKKONEN, Karin. Plot. In: HÜHN, Peter; MEISTER, Jan Christoph; PIER, John; SCHMID, Wolf (Org.). Handbook of Narratology. Berlin; New York: Walter de Gruyter, 2014. p. 706-719.

LAVOCAT, Françoise. Fait et fiction. Pour une frontière. Paris: Seuil, 2016.

MENU, Jean-Christophe. La bande dessinée et son double. Paris: L'Association, 2010

PATRON, Sylvie. Le Narrateur. Paris: Armand Colin, 2009.

PHELAN James. Reading People, Reading Plots: Character, Progression, and the Interpretation of Narrative. Chicago: University of Chicago Press, 1989.

RICOEUR, Paul. Temps et récit I. L'intrigue et le récit historique. Paris: Seuil, 1983.

RYAN, Marie-Laure. Narrative as Virtual Reality. Immersion and Interactivity in Literature and Electronic Media. Baltimore: Johns Hopkins University Press, 2001.

STERNBERG, Meir. Expositional modes and Temporal ordering in Fiction. Bloomington: Indiana University Press, 1993 (1978).

TRUFFAUT, François. Le Cinéma selon Hitchcock. Paris: Seghers, 1975.

VILLENEUVE, Johanne. Le Sens de l'intrigue, ou la narrativité, le jeu et l'invention du diable. Québec: Presses Universitaires de Laval, 2004.

Recebido: 03/01/17

Aprovado: $12 / 03 / 17$

Contato: raphael.baroni@unil.ch 